\title{
Exemplary versus statistical evidence? Response to Berg-Schlosser
}

\author{
CHRISTIAN WELZEL ${ }^{1} \&$ RONALD INGLEHART ${ }^{2}$ \\ ${ }^{1}$ International University Bremen (IUB), Germany; ${ }^{2}$ University of Michigan at Ann \\ Arbor, USA
}

We appreciate Dirk Berg-Schlosser's discussion of our article. He makes a number of positive comments and also expresses some doubts, raising important issues that should be clarified. In this limited space, we will not address all of the diverse matters he discusses, but will concentrate on his major points.

Berg-Schlosser expresses concerns about our measurements, arguing that the indicators we used to measure a society's available resources, its emphasis on emancipative values and its degree of effective democracy are based on 'guesstimates'. This is true in the sense that any measure in the social sciences involves a certain amount of measurement error. Berg-Schlosser's critique, however, is pointless insofar as he fails to provide evidence for his claim that there are better indicators of a society's resources, its emphasis on emancipative values and its degree of effective democracy than the ones we used - and we doubt that he could, because any alternative indicators of which we are aware correlate strongly with the ones we used (usually at $r=0.8$ or $0.9)$.

For example, Berg-Schlosser criticizes our measure of effective democracy, asserting that Singapore would obtain a medium democracy score using this measure. This conclusion is false. We measure effective democracy by combining two separate components. First, we use the Freedom House scores for liberal and political rights as an indicator of the extension of rights. Then we weight the extension of rights by the elite corruption scores provided by Transparency International, using the inverse of these scores as a measure of 'elite integrity' or 'rule of law'. We consider 'elite integrity' as the factor deciding how effective or ineffective a given extension of rights is in light of actual elite behavior. Mathematically, this is expressed by multiplying the extension of rights with the degree of 'elite integrity'. This procedure prevents high levels in one of the two components compensating for low levels in the other, because multiplication is sensitive to discrepancies. Hence, Berg-Schlosser is wrong in claiming that our measure would locate Singapore at a medium level 
of effective democracy. Calculating the product of the two components locates Singapore at the bottom of the democracy scale. This is realistic. With a low extension of civic rights, there can be no democracy, even if the elites are perfectly 'integer' (i.e., uncorrupt).

Berg-Schlosser ignores the fact that the need for measurement accuracy differs between case studies and large-N statistical analyses. Large-N statistical analyses do not require the involved indicators to be completely error-free for each single nation. It is sufficient that the indicators be valid in that they capture the overall pattern of cross-national variation. Whether they do so can be tested by a cross-validation, examining how strongly an indicator correlates with other indicators that are supposed to represent the same dimension. This is exactly what we did. We outlined that available resources, emancipative values and effective democracy each contribute to broadening the scope of 'human choice' that societies offer their constituents. Accordingly, a society's resources, its emphasis on emancipative values and its degree of effective democracy represent the same underlying dimension, which we described as 'human development'. Then we examined the statistical linkages between the indicators that we proposed for measuring available resources, emancipative values and effective democracy. We found very strong linkages, showing that these indicators do indeed reflect a common underlying dimension. Given that these indicators were originally designed to measure different phenomena and have been taken from completely different sources, it is highly unlikely that the strong linkages between them simply reflect measurement error.

Berg-Schlosser's explicit distaste of large-N statistical analyses leads him to unjustified statements. He characterizes as 'superficial' our finding that available resources, emancipative values and effective democracy converge in a common dimension of human development. The fact, however, that this dimension is significantly present across the board, irrespective of the complex specialties of more than 70 diverse nations, is hardly superficial. It is fundamental, indicating an over-arching cross-cultural pattern. Berg-Schlosser's perspective on smaller subsets of peculiar cases blinds this pattern out, but blinding something out is not equal to ruling it out.

Berg-Schlosser raises false expectations concerning general theories. He believes that in order to be general a theory should not allow for exceptional cases. He also uses the term 'probabilistic theory' in a negative sense - implying that theories must be deterministic in order to be falsifiable. Yet probabilistic theories are perfectly falsifiable. We argued that human development is not generated by progress in all of its three components at the same time, but by a causal sequence in which emancipative values operate as a precondition rather than a consequence of effective democracy. We tested this assumption showing that emancipative values in 1990 had a stronger effect 
on temporally subsequent levels of democracy than prior democratic traditions had on self-expression values in 1990 . This test could have also shown the opposite result, falsifying our assumption - but it did not.

Relationships in the social world do not follow iron laws. We have shown that the linkage between emancipative values and effective democracy is strong at the societal level. However, there is a relatively large 'range of uncertainty' within which the linkage is not visible. Not every one-unit increase in emancipative values produces a one-unit increase in effective democracy. Yet a multiple-unit increase in self-expression values, which exceeds the 'range of uncertainty, is almost certainly reflected in an increase in effective democracy. We were able to measure this 'range of uncertainty' by extending the cultural and institutional variation onto a global scale rather than focusing on a narrow subset of exotic cases.

Our theory is not unhistorical, as Berg-Schlosser alleges. He cites Rueschemeyer et al. (1992) as a historical study that falsifies our modernization theory. However, these authors maintained exactly the opposite: modernization is relevant in shaping the preconditions for social movements and class coalitions that aim at establishing political representation and democracy. In addition, Welzel (2002: 235-287) discusses historical evidence showing that the linkage between available resources, a culture that emphasizes human autonomy, and institutions that are based on individual rights is regular and systematic.

We do not question that an exemplary approach provides more casespecific insights. Yet, an exemplary approach has its own limitations. These limitations originate in the restricted case coverage, which makes it impossible to assess how representative one's findings are for all the cases not taken into account. Exemplary evidence provides additional insights, but these insights can neither invalidate nor replace statistical evidence. Large-N statistical evidence is indispensable, since generalization is the very essence of theory building.

\section{References}

Rueschemeyer, D., Huber Stephens, E. \& Stephens, J.D. (1992). Capitalist development and democracy. Chicago, IL: University of Chicago Press.

Welzel, C. (2002). Fluchtpunkt Humanentwicklung. Opladen: Westdeutscher Verlag.

Address for correspondence: Christian Welzel, School of Humanities and Social Sciences, International University Bremen, PO Box 750 561, D-28725 Bremen, Germany

E-mail: c.welzel@iu-bremen.de 